\section{POS0672 FIRST-IN-HUMAN STUDY OF GS-5718, AN ORAL IRAK-4 INHIBITOR, IN HEALTHY SUBJECTS: PHARMACOKINETICS, SAFETY, TOLERABILITY, AND ASSESSMENT OF EFFECT OF FOOD AND ACID REDUCING AGENTS ON EXPOSURE}

\section{K. Anderson ${ }^{1}$, C. H. Hsueh ${ }^{1}$, O. Gurtovaya ${ }^{1}$, A. Mathur ${ }^{1}$, J. Taylor ${ }^{1}$, A. Serone ${ }^{1}$} A. A. Othman ${ }^{1} .{ }^{1}$ Gilead Sciences, Inc., Foster City, United States of America

Background: GS-5718 is a potent and selective interleukin-1 receptor-associated kinase 4 (IRAK4) inhibitor in clinical development for treatment of inflammatory diseases.

Objectives: The aim of this first-in-human study was to evaluate the pharmacokinetics, safety, and tolerability of GS-5718; and the effect of food and acid-reducing agents (ARA) on GS-5718 pharmacokinetics in healthy subjects. Methods: This was a blinded, randomized, placebo-controlled, single and multiple (once daily for 10 days) oral dose study. Healthy male and female subjects were enrolled in ascending dose cohorts and randomized to receive GS-5718 (15, 50 or $150 \mathrm{mg}$ ) or placebo. GS-5718 was administered fasted in the single ascending dose cohorts, and under fed conditions (standard meal) in the multiple dose cohorts. The effects of a high-fat meal and omeprazole (a representative ARA) on GS-5718 $50 \mathrm{mg}$ dose pharmacokinetics were also evaluated. Serial blood samples were collected and GS-5718 pharmacokinetic parameters were characterized. Safety was assessed by review of adverse events (AEs), clinical laboratory tests, and vital signs. Results: A total of 74 subjects ( $n=62$ GS- $5718 ; n=12$ placebo) enrolled and completed study drug treatments in this study. GS-5718 was generally well tolerated at all evaluated dose levels; AEs were mild in severity and no dose-limiting toxicities, serious AEs, nor clinically relevant electrocardiogram or vital sign abnormalities were observed in subjects administered GS-5718. GS-5718 exposure was approximately dose proportional across the evaluated multiple ascending dose range. GS-5718 showed low-to-moderate pharmacokinetic variability with median half-life of 25 to 33 hours and 1.6 to 2.4- fold accumulation at steady-state, which was achieved by Day 5-7 of dosing. Food had no clinically meaningful impact on GS-5718 exposure (AUC and $C_{\max }$ ) at the $50 \mathrm{mg}$ dose. Co-administration of omeprazole with GS-5718 reduced GS-5718 exposure (AUC and $\mathrm{C}_{\text {}}$ ) by $23 \%$ and $43 \%$, respectively, at the $50 \mathrm{mg}$ dose.

Conclusion: GS-5718, administered once daily, was well tolerated following single or multiple dosing up to $150 \mathrm{mg}$. The pharmacokinetic and safety profile of GS-5718 support the further development in inflammatory diseases with oncedaily administrations.

Disclosure of Interests: Kacey Anderson Shareholder of: Gilead Sciences, Employee of: Gilead Sciences, Chia-Hsiang Hsueh Shareholder of: Gilead Sciences, Employee of: Gilead Sciences, Oksana Gurtovaya Shareholder of: Gilead Sciences, Employee of: Gilead Sciences, Anubhav Mathur Shareholder of: Gilead Sciences, Employee of: Gilead Sciences, James Taylor Shareholder of: Gilead Sciences, Employee of: Gilead Sciences, Adrian Serone Shareholder of: Gilead Sciences, Employee of: Gilead Sciences, Ahmed A. Othman Shareholder of: Gilead Sciences, Employee of: Gilead Sciences DOI: 10.1136/annrheumdis-2021-eular.2238

\section{POS0673 TOLEROGENIC DENDRITIC CELLS IN RHEUMATOID ARTHRITIS PATIENTS: NEWS AND PROMISES}

Y. Kurochkina ${ }^{1}$, E. Chernykh ${ }^{2}$, A. Sizikov ${ }^{3} .{ }^{1}$ Research Institute of Clinical and Experimental Lymphology - Branch of the Institute of Cytology and Genetics, Siberian Branch of the Russian Academy of Sciences, Rheumatology, Novosibirsk, Russian Federation; ${ }^{2}$ Federal State Budgetary Scientific Institution Research Institute of Fundamental and Clinical Immunology, Laboratory of Cells Immunotherapy, Novosibirsk, Russian Federation; ${ }^{3}$ Federal State Budgetary Scientific Institution Research Institute of Fundamental and Clinical Immunology, Rheumatology, Novosibirsk, Russian Federation

Background: Dendritic cells (DCs) are known to contribute to the pathogenesis of rheumatoid arthritis (RA) through presentation of cartilage glycoprotein, production of proinflammatory cytokines and activation of Th1/Th17 responses. Along with stimulating activity, DCs may exhibit suppressive functions via capacity to induce $T$ cell apoptosis/anergy and to generate regulatory $T$ cells. Since these DCs have potential to control autoreactive T-lymphocytes, the enhancing of tolerogenic properties of DCs seems to be a new important strategy in treatment of RA. Dexamethasone is widely used in clinical practice and can be used as a tolerogenic substance. Therefore, the properties of DCs generated in presence of dexamethasone are of great clinical interests.

Objectives: The aim of our study is to describe the properties of tolerogenic DCs, generated with dexamethasone in patients with RA and their influence on autologous T-cells.

Methods: Sixty five patients with RA with high and moderate activity of disease were recruited in this study. All patients follow ACR/EULAR criteria (2010). All studies were performed after receiving informed consent. All patients received conventional synthetic DMARDs. DCs were generated from blood monocytes culturing for 5 days with GM-CSF and IFN- $\alpha$ in the presence dexamethasone (dexDCS), applied on third day. LPS as maturation stimuli was added on fourth day. The expression of CD14, CD83, HLA-DR, TLR-2 on the surface of DCs was measured by flow cytometry. The functions of DCs were evaluated by measuring cytokine production and DCs allostimulatory activity in mixed lymphocyte culture. Mature DCs generated in absence of dexamethasone used as control.

Results: We revealed that dexDCs are characterized by enhanced expression of CD14+cells and decreased number of CD83+cells but percent of HLA-DR+cells were constant (about 85). DexDCs show high expression of TLR-2 is seen as tolerogenic molecule $(75 \%$ vs $51 \%, p=0.05$ compared to control). DexDCs also have marked prominent increase of TNF $\alpha / \mathrm{LL}-10$ ratio in contrast to control $(0.59 \mathrm{vs} 1.8$ $\mathrm{p}=0.03)$. DexDCs suppressed proliferation of allogenic T-cells $(2005 \mathrm{vs} 7980 \mathrm{cpm}$ $p=0.0002$ ). To assess the stability of the $D C$ in the proinfflamatory micro-environment after assessing stimulatory activity dexDCs were then cultivated with LPS and allostimulatory activity were evaluated one more. The stimulation activity dexDCs after incubation with LPS were not increase (4692 vs $6053 \mathrm{cpm}, \mathrm{p}=0.7$ ). Also earlier we showed possibility of dexDCs induse apoptosis of autologous T-cells, activation of CD4+IL10+Tr1 and possession of antigen-specific suppression.

Conclusion: The data obtained indicate that dexDCs from RA patients have the main tolerogenic features and stable in inflammatory environment that proves their potential in the treatment of rheumatoid arthritis.

Disclosure of Interests: None declared

DOI: 10.1136/annrheumdis-2021-eular.2261

\begin{tabular}{|l|l}
\hline POS0674 & DIRECT COMPARISON OF EFFECTIVENESS AND \\
SAFETY OF TOFACITINIB AND BARICITINIB IN \\
PATIENTS WITH RHEUMATOID ARTHRITIS IN REAL- \\
WORLD SETTINGS
\end{tabular}

N. Iwamoto ${ }^{1}$, T. Suzuki ${ }^{2}$, A. Okada ${ }^{3}$, K. Fujikawa ${ }^{4}$, T. Aramaki $^{5}$, A. Mizokami ${ }^{4}$, Y. Ueki ${ }^{5}$, A. Kawakami ${ }^{1}{ }^{1}$ Nagasaki University Graduate School of Biomedical Sciences, Department of Immunology and Rheumatology, Division of Advanced Preventive Medical Sciences, Nagasaki University Graduate School of Biomedical Sciences, Nagasaki, Japan, Nagasaki, Japan; ${ }^{2}$ Sasebo City General Hospital, Department of Internal Medicine, Sasebo, Japan; ${ }^{3}$ Nagasaki Atomic Bomb Museum, Department of Rheumatology, Nagasaki, Japan; ${ }^{4} J a p a n$ Community Healthcare Organization, Isahaya General Hospital, Department of Rheumatology, Isahaya, Japan; ${ }^{5}$ Sasebo Chuo Hospital, Department of Rheumatology, Sasebo, Japan

Background: Tofacitinib is a non-selective first-generation JAK inhibitor and baricitinib was approved for the treatment of Rheumatoid arthritis several years after approve of tofacitinib. Randomized controlled trials have shown good treatment response for RA in these two drugs. However, the evaluation of these two drugs in real-world setting have been rarely reported, moreover, until now, no published data of a direct comparison among JAK inhibitors in RA have been available.

Objectives: To compare the efficacy and safety of the JAK inhibitors tofacitinib and baricitinib in patients with rheumatoid arthritis (RA) by using propensity score matching in a real-world setting.

Methods: A total of 242 patients with RA who were treated with tofacitinib $(n=161)$ or baricitinib $(n=81)$ were enrolled. To avoid confounding, we performed propensity score matching based on multiple baseline characteristic variables, and then 80 baricitinib-treated patients and 57 tofacitinib-treated patients were extracted for the direct comparison. A mixed effect model with a repeated measures analysis of variance (ANOVA) was performed to ascertain whether there were significant differences in clinical efficacy between the two treatment groups during the treatment period.Finally, We evaluated the predictive factor of clinical responses by performing univariate and multivariable logistic regression analyses.

Results: The mean delta disease activity scores (DAS)28-ESR from baseline to 6 months were -1.60 (tofacitinib) and -1.46 (baricitinib). The remission rate defined by the DAS28-ESR at 24 weeks were $21.1 \%$ (tofacitinib) and $25.0 \%$ (baricitinib). There was no significant difference in the clinical response between the baricitinib-treated and tofacitinib-treated groups. Although there was no significant difference, the concomitant use of methotrexate (MTX) showed better clinical efficacy in the cases of baricitinib treatment as compared with in the case of tofacitinib treatment. In both groups, the most common AE was herpes zoster infection, and the AE rates were similar between the two groups. However, the predictive factors contributing to clinical response differed. The concomitant use of oral steroid was independently associated with the achievement of DAS-low disease activity in both groups, whereas in the baricitinib group, the number of biological and/or targeted synthetic DMARDs previously used and the DAS28ESR at the time of initiation were associated with DAS-low disease activity. Conclusion: This study indicate that tofacitinib and baricitinib had comparable efficacies and safety profiles in a real-world setting. However, the influence of clinical characteristics on the treatment response differed between these two drugs. Direct comparison between two JAK inhibitors provide useful information to optimal use of JAK inhibitors in real-world settings.

Disclosure of Interests: None declared

DOI: 10.1136/annrheumdis-2021-eular.3469 